\title{
Canadian Urological Association best practice report: Diagnosis and management of radiation induced hemorrhagic cystitis
}

\author{
George Goucher ${ }^{1}$; Fred Saad²; Himu Lukka ${ }^{3}$; Anil Kapoor ${ }^{1}$ \\ ${ }^{1}$ Department of Surgery (Urology) and Oncology, McMaster University, Hamilton, ON, Canada; \\ ${ }^{2}$ Department of GU Oncology, l'Université de Montréal, Montreal, QC, Canada; ${ }^{3}$ Department of \\ Radiation Oncology, McMaster University, Hamilton, ON, Canada
}

Cite as: Can Urol Assoc J 2018 November 22; Epub ahead of print. http://dx.doi.org/10.5489/cuaj.5788

Published online November 22, 2018

\section{Introduction}

\section{Methodology}

A comprehensive review of studies examining radiation induced hemorrhagic cystitis was performed using PubMed, Medline, and the Cochrane Library database. The bibliographies of relevant articles were searched to avoid exclusion of meaningful articles. Where available, focus was given to systematic reviews and comparative studies, however due to a lack of high quality evidence, case series were included and made up the majority of articles included. All articles were reviewed using the evidence-based medicine levels, with a modified Oxford Grading system for recommendations.

\section{Objective}

This Best-Practice Report seeks to inform and educate readers on radiation induced hemorrhagic cystitis (RHC) as well as a survey of commonly used treatment options available and the evidence available to support a given therapeutic choice. The primary outcome of interest was resolution of hematuria; where available the effects of treatment on urinary symptoms are shared. A relative absence of comparative data has prevented previous reviews from providing firm guidelines in the management of RHC and this report relies heavily on retrospective case series with methodological limitations to form recommendations. Individual patient factors, available resources and new research will continue to shape clinical decision-making.

\section{Background}

Hemorrhagic cystitis refers to a collection of symptoms and signs defined by hematuria, lower urinary tract symptoms and cystoscopic findings indicative of underlying urothelial damage. It can have a number underlying causative factors including treatment with chemotherapeutic agents such as cyclophosphamide and concurrent therapies, post-transplant patients, infection mediated as well as treatment 
with radiation therapy. Radiation induced hemorrhagic cystitis (RHC) is often an adverse event in patients with previous radiation to the pelvis for urologic or gynecologic malignancy and will be the focus of this best practice report.

RHC is generally considered a separate pathologic process from acute radiation effects, with early signs of urothelial effects occurring at 3 months and pathological changes occurring 6-12 months after radiation (Marks, Carroll, Dugan, \& Anscher, 1995). New onset symptoms have been recorded up to 20 years after radiation treatment (Levenback, 1994). The estimates for prevalence of RHC after radiation treatment varies widely depending on the diagnostic definitions used, but different studies have quoted them as 9-21\% following treatment of prostate cancer, 3-6.7\% for cervical cancer and 2-47\% for bladder cancer (McAchran \& Rackley, 2008). The use of modern treatment techniques such as intensity-modulated radiation therapy and volumetric modulated arc therapy have reduced the radiation dose to the bladder. Randomized studies have shown that the use of higher doses in the management of prostate cancer have resulted improved PSA control using these modern treatment techniques but with the same incidence of late complications. There has also been a trend to using hypofractionation radiotherapy regimens (higher dose per fraction given over a shorter timeframe). To minimize late bladder effects emphasis needs to be paid to ensuring doses received by radiovulnerable structures such as bowel and bladder are kept within acceptable limits.

The exact mechanism by which radiation causes damage to the bladder is not entirely understood but believed to be multi-factorial. While direct damage to DNA is believed to be rare, it is hypothesized that indirect damage occurs through the creation of free radicals (Cohn, Richards, \& Steinberg, 2015). Histological studies have demonstrated increased proliferation of the urothelium in the months following radiation. Damage to tight cellular junctions and the loss of the normal polysaccharide layer allow for increased permeability of urine bacteria and metabolites causing increased damage to the underlying tissue (Marks et al., 1995).This altered permeability of the urothelial cell layer has been demonstrated to be involved in late stage radiation changes in rat models (Jaal \& Dorr, 2006) and is hypothesized to play a large role in the development of post radiation urinary symptoms (C. L. Parsons, 2011)

Diffuse mucosal edema is noted in biopsies taken immediately post radiation. This is followed by development of vascular telangiectasia, sub-mucosal hemorrhage and interstitial fibrosis. Sub-endothelial proliferation, edema and medial thickening may progressively deplete the blood supply to urothelium resulting in endarteritis obliterans causing acute and chronic ischemia (McAchran \& Rackley, 2008).These ischemic and necrotic changes are proposed to give rise to subsequent development of revascularization with superficial, fragile vessels that are responsible for bleeding in radiation cystitis (Cohn et al., 2015). 
Hospitalizations for radiation induced hemorrhagic cystitis can be lengthy and costly. A recent retrospective study assessing 1,111 patients admitted for RHC in 2013 showed the median cost associated with each admission to be $\$ 7,157$ (USD) . This number rose to $\$ 11,100$ for those with hematuria severe enough to merit endoscopic evaluation/treatment (Kiechle et al., 2016). Multiple studies have demonstrated the significant effect its protracted and recurrent nature can have on patient-rated quality of life scales (Droupy, 2017). There exists little consensus of how to best treat RHC, and previous surveys of practicing urologist have shown a lack of awareness of treatment options available (Bahl, 2013).

\section{Classifications}

The EORTC/RTOG classification of late radiation effects is a commonly used classification system for grading of radiation induced hemorrhagic cystitis (J. D. Cox, Stetz, \& Pajak, 1995). It describes a combination of clinical and cystoscopic criteria for reporting late radiation effects. The Lent/SOMA scale has also been developed and uses a more complex combination of subjective, objective, management and analytical factors into radiation effect classification (Anacak, Yalman, Özsaran, \& Haydaroğlu, 2001). A more recent move to replace these systems with a common terminology has led to the development of the Common Terminology Criteria for Adverse Events (Health, 2017) as a uniform lexicon for description of cancer related adverse effects.

\section{Initial management}

\section{Diagnosis and early assessment}

In patients presenting with hematuria post radiation, a thorough assessment is needed to rule out secondary causes before a diagnosis of RHC can be made. In a study exploring cystoscopic evaluation of 185 men treated with brachytherapy for prostate cancer who presented with either macroscopic hematuria, microscopic hematuria or persistent lower urinary tract, 9.6\% were found to have a new bladder tumour compared to 7\% who were found to have radiation cystitis (Leapman, Stock, Stone, \& Hall, 2014). While the majority of these symptomatic post-brachytherapy patients had cystoscopies reported as normal (63.8\%) a clinically significant number did have an observable etiology. Assessment should begin with a detailed history characterizing the symptoms and confirming the history and treatment plan of a patient's radiation therapy. Physical exams including an abdominal and pelvic exam to assess for alternative causes of bleeding should be included. Laboratory tests including a CBC, coagulation studies, serum creatinine, urinalyses, urine culture and cytology should be initiated. As with any patient presenting with hematuria and a high risk of malignancy all patients should undergo axial imaging, preferably a CT-urogram to assess for upper tract sources of bleeding and should also undergo cystoscopic evaluation and biopsies of lesions concerning for malignancy. Mild symptoms may resolve with continuous bladder irrigation with saline solution and this should be tried first in all patients with hematuria associated with clotting or retention. 
Recommendation: Assessment of a patient complaining of hematuria post radiation therapy should identify or exclude other pathologic factors that may explain or contribute to the patient's symptoms (Grade 4C).

\section{Cystoscopic evaluation}

Cystoscopy in patients with new-onset or suspected RHC can be both diagnostic and therapeutic. The appearance of diffuse erythema, telangiectasia with or without ulcerations can help confirm the diagnosis of RHC. At the time of cystoscopy fulguration of suspected vascular lesions may provide control of hematuria. In a case series assessing the effectiveness of cystoscopy and fulguration for hematuria control in patients with either radiation or chemotherapy induced symptoms 61\% (20/33) of patients achieved resolution of symptoms after initial endoscopic treatment(Kaplan \& Wolf, 2009). Only 36\% (4/11) of patients who had refractory symptoms responded to a second cystoscopy and fulguration.

Multiple small case series have assessed alternatives to fulguration with Greenlight Laser, KTP laser treatment and Argon Beam Coagulator (Margreiter, Sharma, Schauer, \& Klingler, 2013; Talab, McDougal, Wu, \& Tabatabaei, 2014; Wines \& Lynch, 2006). All series report laser therapy being well tolerated and having a beneficial effect. In the future they may play a larger role in the treatment of RHC however until larger studies can confirm their safety and effectiveness they remain experimental in nature. Additional studies have investigated the use of intravesical hemostatic gelatin matrix (Floseal) in 6 patients and was noted to be beneficial (Margreiter et al., 2013; Talab et al., 2014; Wines \& Lynch, 2006), fibrin glue has been trialed in the treatment of post hematologic stem cell transplant patients and found to reduce hematuria, (Tirindelli et al., 2014) but it has yet to be investigated in the radiation induced hemorrhagic cystitis population beyond individual case studies (Ouwenga, Langston, \& Campbell, 2004)

Recommendation: All patients with hematuria should undergo at least one initial cystoscopy with or without fulguration of suspect lesions and biopsy of any lesions concerning for malignancy for diagnostic and therapeutic purposes (Grade 3C). Laser therapy and endoscopic use of hemostatic agents may play a role in early management of RHC but insufficient data exists to evaluate their efficacy, safety and long-term follow-up are needed prior to any recommendations being made (Grade $3 D$ ).

\section{Persistent or recurrent clinically significant hematuria}

Intravesical agents

\section{Alum irrigation}

Intravesical aluminous salts act as an astringent agent and exert their effect through protein precipitation causing decreased capillary permeability, contraction of intercellular space, vasoconstriction and hardening of the capillary endothelium 
(Choong, Walkden, \& Kirby, 2000). It is typically administered as a $1 \%$ concentration of alum mixed with sterile water, irrigated through the bladder at 250 - $300 \mathrm{mls}$ per hour, at a duration up to the discretion of the observing clinician. Ideally the bladder should be irrigated free of clots prior to initiation of therapy. Historical case series have demonstrated the efficacy and tolerability of intravesical alum for a wide range of etiologies and presentations of intractable bladder hemorrhages (Goel, 1985; Takashi, Kondo, Kato, Murase, \& Miyake, 1988). The common side effects are bladder spasms, supra-pubic discomfort and clotting of the catheter due to precipitant formation. Individual case studies have been have reported a risk of aluminum toxicity in individuals in renal failure (Phelps, Naylor, Brien, Wilbur, \& Haqqie, 1999).

The most recent, and largest case series reported the use of alum irrigation in 40 patients (38 of whom had previous radiation treatment) with symptoms of hemorrhagic cystitis (Westerman, Boorjian, \& Linder, 2016) . 60\% of these patients demonstrated an improvement in their symptoms and required no further treatment prior to discharge. The treatment was well tolerated and $90 \%$ of patients were able to receive their treatment as prescribed, 2 patients discontinued due to inability to tolerate bladder spasms and 2 for altered LOC. Overall 35\% of patients experienced bladder spasms. Asymptomatic elevation in serum aluminum was detected in 1 patient that resolved on discontinuation of the treatment. At a median follow up of 16.5 months only $32 \%$ of patients required no further treatment, bringing the durability of this treatment into question.

Recommendations: Irrigation with alum is a practical and easily applied treatment for radiation induced hemorrhagic cystitis with a comparatively acute onset of action that is generally well tolerated. Special caution should be used in patients with poor renal function (Grade 3C).

\section{$\underline{\text { Hyaluronic acid }}$}

Hyaluronic acid (HA) is a mucopolysaccharide whose therapeutic benefit is believed to be mediated by the repair of the normal glycoaminoglycan layer of the bladder when applied as intravesical installations. It has been shown that radiation leads to defects in this protective layer leading to chronic inflammatory changes and delayed or prevented healing of urothelial cells (Lazzeri et al., 2016). Its use as an intravesical agent is well studied in the treatment of interstitial cystitis and painful bladder syndrome, where it was recently recommended as a treatment option in the CUA guidelines (A. Cox et al., 2016)

One of the few randomized trials comparing treatment modalities for RHC randomized 36 patients to either intra-vesical therapy with HA or treatment with hyperbaric oxygen therapy (Shao, Lu, \& Shen, 2012). In the HA group patients received intravesical therapy once weekly for 1 month and then monthly for 2 months, patients in the HBOT group received daily treatments for 30 treatments. Follow up assessment of symptoms were done at the 6, 12 and 18 months following therapy. Complete resolution of hematuria was noted in $88 \%, 75 \%$ and $50 \%$ of HA patients at 
those intervals and in $75 \%, 50 \%$ and $45 \%$ of patients in the HBOT group. The difference was not statistically significant at any interval and the author concluded the HA therapy was at least as effective as HBOT in treatment of hematuria.

A prospective observational study of 20 patients undergoing treatment with intravesical HA compared grading of radiation cystitis, including hematuria as per EORTC/ROTG classification (Scale 1-5) before treatment and again three months after the completion of 6 scheduled treatments (Vasssilis, Eftychia, Ivelina, Nikolaos, \& Argyris, 2014). The mean score of radio-cystitis before and after HA treatment was 2.70 and 1.45 respectively $(\mathrm{P}<0.01)$ with only minor side effects described. It is notable that no patients with grade 4 or 5 toxicity, considered severe symptoms, were included in this study. Beyond hematuria, recent prospectively collected data suggests that intravesical treatment with hyaluronic acid in combination with chondroitin sulfate (another glycosamniglycan) produces a clinically significant improvement in post-radiation LUTS and bother. In a study published by Gacci et al. (2016) 80 patients with previous radiation therapy and new onset or worsening LUTS symptoms were shown to have a statistically significant reduction in urgency, frequency, nocturia and bladder pain as measured by patient reported outcomes after intravesical treatment with hyaluronic acid with chondroitin sulfate.

Recommendation: Intravesical therapy with hyaluronic acid may improve symptoms of RHC, and may provide further benefit in those with significant LUTS. Its slow onset of action and lack of research in severe hematuria may limit its usefulness in the acute or in-patient setting (Grade 3C).

Other intravesical agents

Intravesical instillation of the antifibrinolytic agent epsilon aminocaproic acid (EACA), was shown to improve hematuria in 34 patients in case series of 37 patients with intractable bladder hemorrhage, most of whom had either radiation or cyclophosphamide induced cystitis, however this has not been repeated in contemporary studies since its publication in 1992 (Singh \& Laungani, 1992). Intravesical instillations of prostaglandins has been demonstrated in several smaller case series to be effective in treating hemorrhagic cystitis secondary to treatment with cyclophosphamide, but there are no large studies to suggest its effectiveness in radiation induced hemorrhagic cystitis (Levine \& Jarrard, 1993). Silver nitrate instillations have been tried and were found to be ineffective in limiting RHC (Montgomery, Boorjian, Ziegelmann, Joyce, \& Linder, 2016).

Recommendation: Several intravesical options have been trialed in limited case series, but require replication, etiology specific assessment or comparative data before they can be formally included as recommendations (Grade 3D). 


\section{Systemic agents}

\section{Hyperbaric oxygen therapy}

Radiation to the bladder causes obliterative endarteritis of blood vessels creating cellular hypoxia, bladder ischemia and fibrosis. This may lead to superficial fragile blood vessels prone to recurrent bleeding (McAchran \& Rackley, 2008). Patients who undergo hyperbaric oxygenation therapy (HBOT) inhale 100\% O2 at pressures of 1.4 - 3.0ATM allowing for hyperoxygenation of tissue. Hyperoxia induces primary neovascularization , secondary growth of healthy granulation tissue and induces short term vasoconstriction that may help control active bleeding (Capelli-Schellpfeffer \& Gerber, 1999).

HBOT is the most studied treatment option for radiation induced hemorrhagic cystitis. A recent scoping review and meta-analyses evaluating this treatment on resolution of hematuria was published, it included one small randomized control trial, two prospective trials and eleven retrospective case series (Cardinal et al., 2018). This meta-analysis represents 602 patients followed for at least 1 year. Partial or complete response was noted in $84 \%$ of patients (CI 76 - 91) in the pooled analysis. The most common complications were barotrauma, frequently barotraumatic otitis in $6 \%$ of participants. Visual field disturbances were noted in $1 \%$ of participants.

Select case series offer follow up data available for up to a decade after treatment and seem to suggest that successful treatment with HBOT may offer a durable response for patients. One study followed 32 of its participants for an average of 11.6 years and found the resolution rate of macroscopic hematuria to be $81 \%$ (Nakada et al., 2012). A smaller case series supported this data as 12 of 13 participants remained free of hematuria at 10 years (Weiss, Mattei, Neville, \& Hanno, 1994). Recently authors have sought to describe their experience using HBOT as a primary therapy in cases of severe RHC. In one study 38 patients presenting with Grade IV EORTC hematuria requiring transfusions HBOT therapy was used as firstline monotherapy (Dellis, Papatsoris, Kalentzos, Deliveliotis, \& Skolarikos, 2017). After an average of 33 treatments, $87 \%$ of patients had complete resolution of hematuria, long term data was not available to assess the durability to of treatment.

Recommendation: Multiple studies have demonstrated that HBOT is safe and effective and should be considered an early treatment option for RHC in patients who have failed cystoscopy and fulguration. Due to significant resource and expertise requirements, its use may be limited based on access and availability (Grade 3C).

\section{$\underline{\text { Sodium pentosan polysulfate }}$}

Sodium pentosan polysulfate is a semi-synthetic polyscaccharide formulated as a oral medication that serves as a synthetic glycosaminoglycan. It adheres to the bladder mucosa where it supplements the bladder's own glycosaminoglycan layer in a similar fashion as intravesical treatment with hyaluronic acid. It has been previously shown to be effective in the treatment of interstitial cystitis (Hwang, Auclair, Beechinor, \& 
Einarson, 1997). The largest study to assess its effectiveness in hematuria secondary to radiation followed 60 consecutively enrolled patients who were treated with 100 mg PO of SPP T.I.D. (Sandhu, Goldstraw, \& Woodhouse, 2004). In 10 patients hematuria was noted to have resolved completely and there was partial resolution in 21 patients. However the applicability of this study was limited by the large number of participants not available for follow up or who passed away during the study from causes unrelated to hematuria. Two other small case series have also demonstrated the benefits of oral SPP (Hampson \& Woodhouse, 1994) (C.L. Parsons, 1986). Its usefulness was noted primarily by its safety, tolerability and ease of administration, however the onset of action was found to be 1-8 weeks limiting its use in the acute setting. A recent case series has suggested a risk of pigmentary maculopathy associated with long-term chronic use of pentosan polysulfate (median duration of 186 months) (Pearce, Chen \& Jain, 2018)

Recommendation: Several case series have shown a potential reduction of hematuria with treatment with sodium pentosan polysulfate in patients with RHC. It is safe and generally well tolerated, the slow onset of action may limit its usefulness in treatment of acute or severe RHC (Grade 3C).

Experimental options

WF10, an I.V. administered macrophage regulator has shown promising results for treatment and decreased recurrence of radiation induced hemorrhagic cystitis in two studies published at a single center (Veerasarn, Boonnuch, \& Kakanaporn, 2006; Veerasarn et al., 2004), but is not commercially available in Canada. Two small case studies explored the use of estrogen in treatment of hemorrhagic cystitis of multiple etiologies (Liu et al., 1990) (Miller, Burfield, \& Moretti, 1994), while both showed reduction in hematuria, larger studies are needed before recommendations regarding effectiveness or safety can be made. There is conflicting evidence that may point to a role in cranberry juice in preventing acute urinary symptoms in patients undergoing pelvic radiation, however there is no data to suggest it has a role in management of late-effect radiation induced hematuria (Bonetta \& Di Pierro, 2012; Cowan et al., 2012; Hamilton, Bennett, Purdie, \& Herst, 2015)

There is a growing body of evidence exploring the use of tranexamic acid the control of hemorrhage of multiple etiologies. Its use has previously been suggested in the management of RHC (Browne et al., 2015) A randomized control trial recently assessed the use of IV tranexamic acid in controlling of hematuria of multiple etiologies in the acute setting. It found a decreased time on C.B.I. until resolution of hematuria, however no significant difference in blood loss or transfusions rates were noticed in the treatment group (Moharamzadeh, Ojaghihaghighi, Amjadi, Rahmani, \& Farjamnia, 2017). While further studies may demonstrate a benefit in treatment of patients with RHC with tranexamic acid at this time there is insufficient published data to support this. 


\begin{abstract}
Recommendations: Multiple systemic agents have been used in small experimental situations for treatment of radiation induced hemorrhagic cystitis, until further studies are completed no formal recommendations can be made regarding their use (Grade 3D).
\end{abstract}

\title{
Refractory and life-threatening hematuria
}

\section{Trans-arterial embolization}

Advances in interventional radiology have allowed for accurate selective and superselective trans-arterial embolizations (TAE) that provide clinicians management alternatives to more radical surgical procedures in patients with persistent or life threatening hematuria. The benefit of TAE is its safety and tolerability in comparison to more aggressive surgical procedures in the frail and elderly patient who make up a significant proportion of patients with RHC. However these procedures are not without their own risks and side effects.

Unfortunately many of the studies on TAE for hematuria include urologic bleeding of multiple etiologies and do not discriminate between blood loss from bladder or prostatic origins. In a case series of 44 patients looking at the role of TAE in management of intractable hematuria hemorrhage of oncologic origin, Ligori et al. found that the majority of patients experienced an initial resolution of hematuria (82\%) (Liguori et al., 2010) . The ability to perform selective and super-selective has reduced the risk of ischemic related side effects. Historical studies have demonstrated ischemic mediated side effects in as high as 65\% in patients undergoing internal iliac artery embolization (Pisco, Martins, \& Correia, 1989)although most of these are transient post-embolization syndrome they also include more serious complications like bladder necrosis and in rare cases Brown-Sequard syndrome. Comparable modern series published in the last decade quote a complication rates from 9 - 31\% that were usually minor and self-resolving, with a technical success rate of 88-100\% (Korkmaz et al., 2016), (Loffroy et al., 2014).

Recommendation: Transarterial embolization is a viable option for control of radiation induced hematuria in those whom less invasive methods have been unsuccessful. Preference should be given to selective, or superselective embolization when available to lessen possible side effects (Grade $3 C$ ).

\section{Formalin}

Intravesical formalin was first described in the treatment of bladder hemorrhage in the late 1960's, and was soon assessed in the radiation induced hematuria cohort. The proposed mechanism involved capillary occlusion and protein fixation at the urothelium level (Ziegelmann, Boorjian, Joyce, Montgomery, \& Linder, 2017)Due to pain with administration it must administered in an OR setting with either a general or spinal anesthetic. While its rapid onset of action is appealing, its use is somewhat controversial because of its high morbidity which appears to be proportional to the concentration of formalin used (McAchran \& Rackley, 2008). 
The largest review of the efficacy of formalin instillation from treating hemorrhagic cystitis of multiple etiologies was a systematic review of retrospective case series published in 1989 (Donahue \& Frank, 1989). The article included 235 patients stratified into three groups by the concentration of formalin instilled (10\% vs. $3-6 \%$ vs. $1-2 \%)$. The complete response rates were 88,78 and $71 \%$ respectively. One benefit of formalin instillations was that complete response was typically achieved within 48 hours of a single instillation. Major complications were typically associated with refluxing into the upper urinary tract and consisted of ureteric stricture function, UPJ and UVJ obstruction requiring urinary diversion, decreased bladder capacity and vesicular fistulas. The mortality rates for $10 \%$ formalin instillation was recorded at $5.7 \%$ and $2.2 \%$ for the $4-6 \%$ concentration group, no mortalities occurred in the 21 patients treated with $1-2 \%$ concentration.

Several other studies were completed that found formalin to be associated with a high treatment efficacy but with potentially severe complications. In a study of 35 patient with RHC post cervical radiation, $89 \%$ were found to have complete response after a single instillations however 31\% had major complications (Dewan, 1993). The one contemporary study investigated 8 patients treated with formalin instillations after less invasive treatments had failed, in this study each patient had a preoperative cystogram to assess for perforation or VUR, and if any reflux was suspected Fogarty catheters were used to obstruct the ureter (Ziegelmann et al., 2017). Formalin concentration ranged from 1-4\% and contact time was kept to 10 - 15 minutes. The complete resolution rate was $75 \%$ and only 1 patient had major complications necessitating ICU admission. A detailed description of the procedure can be found within this article for clinicians unfamiliar with this treatment option.

Procedural variations for decreasing the morbidity associated with formalin instillations have been proposed; from decreasing formalin concentration to alternative methods of formalin delivery. A small prospective study compared intravesical instillation of $4 \%$ formalin in 11 patients to endoscopic placement of formalin soaked pledgets (Lojanapiwat, Sripralakrit, Soonthornphan, \& Wudhikarn, 2002). Success rate was similar ( $82 \%$ and $75 \%$ respectively), however the intravesicle instillation group suffered from four major complications, whereas the pledget group suffered only minor side effects. The differences were not statistically significant and while a comparable method was described in two individual case studies (Haraguchi, 2007; Lowe \& Stamey, 1997)follow-up with further larger or prospective studies is lacking.

Recommendation: Due to significant morbidity associated with the procedure, Formalin instillations should only be used in those who have failed less invasive treatments. If treatment is necessary, all attempts should be made to prevent reflux into the upper tracts and the patient needs careful monitoring for potential side effects (Grade $3 C$ ). 
Cystectomy and urinary diversion

Unfortunately a small percentage of patients will present with life threatening hemorrhagic cystitis that is refractory to conservative and non-operative measures. These patients can be successfully treated with urinary diversion and cystectomy, however the associated morbidity with this procedure is high. Several case series have examined the use of cystectomy in patients who had previously failed less invasive therapies. One series identified 21 patients with hemorrhagic cystitis, 17 from radiation therapy, who underwent cystectomy (Linder, Tarrell, \& Boorjian, 2014). In this series $42 \%$ of patients experienced severe complications (defined as Clavien grade III-V ) and the 90 day mortality rate was $16 \%$. This study echoes the findings of a larger case series where surgical outcomes from men undergoing urinary diversion via cystectomy for multiple adverse effects of radiation including hematuria (Bassett et al., 2017). In this series $36 \%$ of patients experienced Clavian III or greater complications, including $15 \%$ who required a second operation and death in $4.5 \%$ of patients within the first 90 days. These rates of complications outpace the comparable rates in patients undergoing radical cystectomy for bladder cancer, both authors attributed this to baseline fragility and comorbidities in this patient population exacerbated with the challenge of operating in a previously radiated field. Small case studies have evaluated the use of urinary diversion alone in those who may not tolerate a cystectomy, either through cutaneous ureterostomy (Pomer, Karcher, \& Simon, 1983)or bilateral nephrostomy tubes (Zagoria, Hodge, Dyer, \& Routh, 1993). Although both studies were quite small they showed improvement in hematuria and may be a beneficial surgical option in patients whom cystectomy is not a viable option. Caution should be advised, as long term follow-up in urinary diversion without cystectomy for benign conditions have shown a high rate of complications in the remaining bladder (Fazili, Bhat, Masood, Palmer, \& Mufti, 2006).

Recommendation: Urinary diversion with or without cystectomy for RHC should be reserved only for those who have failed previously available therapy, and clinicians and patients should both be aware of the high morbidity and mortality of the procedure before proceeding with surgery (Grade $3 C$ ).

\section{Discussion}

\section{Treatment sequencing}

There is a lack of consensus or comparative evidence to suggest superiority of one treatment of RHC over another. The lack of high quality evidence has limited the ability for previous authors to conclusively state the order that treatment should be provided in the cases of refractory therapy. There have been several previous attempts to make recommendations for treatment algorithms, with the general consensus that treatments should be offered initially using the least invasive approaches and progressing to more invasive approaches as a general principle (Alesawi, El-Hakim, Zorn, \& Saad, 2014; Liem, Saad, \& Delouya, 2015; Moro, Junior, Riccetto, \& Palma, 2015; Smit \& Heyn, 2010; Thompson, 2013). 
This report seeks to provide a logical and stepwise approach to the management of radiation cystitis. Given the wide variety of clinical states that a patient with RHC may present, a linear treatment algorithm would be insufficient to provide treatment suggestions appropriate for different patients depending on the acuity and severity of the symptoms. Instead we have divided our treatments into three groups based on severity of clinical presentation and associated morbidity with treatment options (Table 2). The first box represents initial management and provides suggestions for investigations and supportive therapy. It is focused on upfront investigations to rule out other causes that may explain or exacerbate hemorrhagic cystitis. Underlying correctable factors such as infection, malignancy or coagulopathies should be treated if clinically possible.

The second group; patients with persistent or recurrent clinically significant hematuria may apply to those who have required CBI, irrigation and clot evacuation as an in-patient and continue to have hematuria but also acknowledges that there are many patients who have recurrent hematuria that is clinically significant who may benefit from treatment as an outpatient. The definition of "clinically significant" here may be broadly applied to any gross hematuria that is causing a marked burden on a patient or negatively impacting their day-to-day living. Treatments recommended for patients in this group have been shown to be both effective, safe and well tolerated by most patients. The four recommended treatment options here were chosen because they have evidence replicated from multiple centers, are clinically available in Canada and have been approved and proven in comparable pathologies. As more research continues to be done on radiation cystitis we may be able to add further therapies to this group. Hyperbaric oxygen treatment is offered here as initial therapy to reflect the growing amount of evidence that suggest it is an effective and safe treatment for RHC including those presenting with high grade toxicities. Within this group a clinician may consider onset of action as a deciding factor between therapies, as only alum irrigation has been shown to provide improvement within days of being started. Hyaluronic acid and sodium pentosan polysulfate both treat urinary symptoms associated with hemorrhagic cystitis in addition to hematuria.

The third category, "Refractory and Life-threatening hematuria" includes patients who have previously been trialed and failed with treatment options from group 1 and group 2 and continue to have symptomatic hematuria that poses a threat to their continued safety and well-being. In these patients treatment options typically have a rapid onset of action and have been proven to be effective but may be associated with significant morbidity to the patient. Less invasive procedures such as embolization or formalin instillation should be attempted prior to surgical therapy. Once a mainstay of treatment, and commonly recommended in many former treatment algorithms, we recommend caution with the usage of formalin instillations with special care to limit contact time, use the least concentrated formulation necessary and to prevent systemic uptake or refluxing into the upper tracts. Clinicians 
and patients need to be aware of the high risk associated with cystectomy in postradiation patients before proceeding to surgical intervention. In patients whom a cystectomy would not be appropriate, or with a limited life span, temporary diversion with either percutaneous nephrostomy tubes or cutaneous ureterostomies may be considered as an alternative.

\section{Conclusion}

Radiation induced hemorrhagic cystitis remains a challenging medical condition that often presents in a frail and vulnerable population. In this report we have presented a general approach and suggestions for management as well as an overview of key research supporting specific therapies. These guidelines should always be used in the context of an individual patients presentation, and may continue to evolve, as more evidence is available on the subject. 


\section{References}

1. Alesawi, A. M., El-Hakim, A., Zorn, K. C., \& Saad, F. (2014). Radiationinduced hemorrhagic cystitis. Curr Opin Support Palliat Care, 8(3), 235-240. doi:10.1097/SPC.0000000000000073

2. Anacak, A., Yalman, D., Özsaran, Z., \& Haydaroğlu, H. (2001). Late radiation effects to the rectum and bladder in gynecologic cancer patients: the comparison of LENT/SOMA and RTOG/EORTC late-effects scoring systems. International Journal of Radiation Oncology,Biology, Physics, 50(5), 11071112.

3. Bahl, A. (2013). Current management and proposal for guidance of radiation cystitis (RC): Results of a British Uro-oncology Group (BUG) survey. Paper presented at the ASCO: Genitourinary Cancers Symposium, Orlando.

4. Bassett, M. R., Santiago-Lastra, Y., Stoffel, J. T., Goldfarb, R., Elliott, S. P., Pate, S. C., . . Urologic Reconstructive Network of, S. (2017). Urinary Diversion for Severe Urinary Adverse Events of Prostate Radiation: Results from a Multi-Institutional Study. The Journal of Urologyl, 197(3 Pt 1), 744750. doi:10.1016/j.juro.2016.10.091

5. Bonetta, A., \& Di Pierro, F. (2012). Enteric-coated, highly standardized cranberry extract reduces risk of UTIs and urinary symptoms during radiotherapy for prostate carcinoma. Cancer Manag Res, 4, 281-286. doi:10.2147/CMAR.S35342

6. Browne, C., Davis, N. F., Mac Craith, E., Lennon, G. M., Mulvin, D. W., Quinlan, D. M., ... Galvin, D. J. (2015). A Narrative Review on the Pathophysiology and Management for Radiation Cystitis. Adv Urol, 2015, 346812. doi:10.1155/2015/34681

7. Capelli-Schellpfeffer, M., \& Gerber, G. S. (1999). The Use of Hyperbaric Oxygen in Urology. The Journal of Urology

8. , 162(3), 647-654.

9. Cardinal, J., Slade, A., McFarland, M., Keihani, S., Hotaling, J. N., \& Myers, J. B. (2018). Scoping Review and Meta-analysis of Hyperbaric Oxygen Therapy for Radiation-Induced Hemorrhagic Cystitis. Curr Urol Rep, 19(6), 38. doi:10.1007/s11934-018-0790-3

10. Choong, S. K. S., Walkden, M., \& Kirby, R. (2000). The management of intractable haematuria. BJU Int, 86, 951-956.

11. Cohn, J., Richards, K., \& Steinberg, G. (2015). The Management of Radiation Cystitis. In E. Ehrenpreis, R. Marsh, \& W. Small (Eds.), Radiation Therapy for Pelvic Malignancy and its consequences. New York, NY: Springer.

12. Cowan, C. C., Hutchison, C., Cole, T., Barry, S. J., Paul, J., Reed, N. S., \& Russell, J. M. (2012). A randomised double-blind placebo-controlled trial to determine the effect of cranberry juice on decreasing the incidence of urinary symptoms and urinary tract infections in patients undergoing radiotherapy for cancer of the bladder or cervix. Clin Oncol (R Coll Radiol), 24(2), e31-38. doi:10.1016/j.clon.2011.05.009

13. Cox, A., Golda, N., Nadeau, G., Curtis Nickel, J., Carr, L., Corcos, J., \& Teichman, J. (2016). CUA guideline: Diagnosis and treatment of interstitial cystitis/bladder pain syndrome. Can Urol Assoc J, 10(5-6), E136-E155. doi:10.5489/cuaj.3786 
14. Cox, J. D., Stetz, J., \& Pajak, T. F. (1995). Toxicity criteria of the radiation therapy oncology group (RTOG) and the European organization for research and treatment of cancer (EORTC). The International Journal of Radiation Oncology, Biology, Physics, 31(5), 1341-1345.

15. Dellis, A., Papatsoris, A., Kalentzos, V., Deliveliotis, C., \& Skolarikos, A. (2017). Hyberbaric oxygen as sole treatment for severe radiation - induced haemorrhagic cystitis. Int Braz J Urol, 43(3), 489-495. doi:10.1590/S16775538.IBJU.2016.0451

16. Dewan, A. K. M., G.M.; Ravi, R.; . (1993). Intravesical formalin for hemorrhagic cystitis following irradiation of cancer of the cervix.. International Journal of Gynaecology and Obstetrics, 42(2), 131-135.

17. Donahue, L., \& Frank, I. (1989). Intravesical formalin for hemorrhagic cystitis: analysis of therapy. The Journal of Urology, 141(4), 809-812.

18. Droupy, S. (2017). The therapeutic approach to different forms of cystitis: impact on public health. Urologia, 84(Suppl 1), 8-15. doi:10.5301/uj.5000262

19. Fazili, T., Bhat, T. R., Masood, S., Palmer, J. H., \& Mufti, G. R. (2006). Fate of the leftover bladder after supravesical urinary diversion for benign disease. The Journal of Urology, 176(2), 620-621. doi:10.1016/j.juro.2006.03.056

20. Gacci, M., Saleh, O., Giannessi, C., Chini, T., Della Camera, P. A., Detti, B., . . . Serni, S. (2016). Bladder Instillation Therapy With Hyaluronic Acid and Chondroitin Sulfate Improves Symptoms of Postradiation Cystitis: Prospective Pilot Study. Clin Genitourin Cancer, 14(5), 444-449. doi:10.1016/j.clgc.2016.01.016

21. Goel, A. K. R., M.S.; Bhagwat, A.G.; Vaidyanathan, S.; Goswami, A.K.; Sen, T.K.;. (1985). Intravesical irrigation with alum for the control of massive bladder hemorrhage.. The Journal of Urology, 133(6), 956-957.

22. Hamilton, K., Bennett, N. C., Purdie, G., \& Herst, P. M. (2015). Standardized cranberry capsules for radiation cystitis in prostate cancer patients in New Zealand: a randomized double blinded, placebo controlled pilot study. Support Care Cancer, 23(1), 95-102. doi:10.1007/s00520-014-2335-8

23. Hampson, S. J., \& Woodhouse, C. R. (1994). Sodium Pentosanpolysulphate in the management of haemorrhagic cystitis: experience with 14 patients. . European Urology, 25(1), 40-42.

24. Haraguchi, T. Y., T.; Takechi, Y.;. (2007). Hemorrhagic radiation cystitis: a transurethral treatment with formalin-soaked pledgets]. Hinyokika Kiyo, 53(8), 585-587.

25. Health, N. I. o. (2017). Common Terminology Criteria for Adverse Events (CTCAE) v5.0.

26. Hwang, P., Auclair, B., Beechinor, D. D., M., \& Einarson, T. R. (1997). Efficacy of pentosan polysulfate in the treatment of interstitial cystitis: a metaanalysis. Urology, 50(1), 39-43.

27. Jaal, J., \& Dorr, W. (2006). Radiation-induced damage to mouse urothelial barrier. Radiother Oncol, 80(2), 250-256. doi:10.1016/j.radonc.2006.07.015

28. Kaplan, J. R., \& Wolf, J. S., Jr. (2009). Efficacy and survival associated with cystoscopy and clot evacuation for radiation or cyclophosphamide induced hemorrhagic cystitis. The Journal of Urology, 181(2), 641-646. doi:10.1016/j.juro.2008.10.037

29. Kiechle, J. E., Kim, S. P., Yu, J. B., Maurice, M. J., Dong, S., Cherullo, E. E., \& Abouassaly, R. (2016). Economic Burden Associated with Hospitalization 
for Radiation Cystitis: Results from a Statewide Inpatient Database. Urology Practice, 3(6), 437-442. doi:10.1016/j.urpr.2015.10.007

30. Korkmaz, M., Sanal, B., Aras, B., Bozkaya, H., Cinar, C., Guneyli, S., . . . Oran, I. (2016). The short- and long-term effectiveness of transcatheter arterial embolization in patients with intractable hematuria. Diagn Interv Imaging, 97(2), 197-201. doi:10.1016/j.diii.2015.06.020

31. Lazzeri, M. L., Hurle, R., Casale, P., Buffi, N. M., Lughezzani, G., Fiorini, G., ... Barbagli, G. (2016). Managing chronic bladder diseases with the administration of exogenous glycosaminoglycans: an update on the evidence. Therapeutic advances in urology, 8(2), 91-99. doi:10.1177/

32. Leapman, M. S., Stock, R. G., Stone, N. N., \& Hall, S. J. (2014). Findings at cystoscopy performed for cause after prostate brachytherapy. Urology, 83(6), 1350-1355. doi:10.1016/j.urology.2014.01.031

33. Levenback, C. E., P.; Burke, T; Morris, M; Gershenson, D. (1994). Hemorrhagic Cystitis Following Radiotherapy for Stage 1B Cancer of the Cervix. Gynecologic Oncology, 55, 206-210.

34. Levine, L. A., \& Jarrard, D. F. (1993). Treatment of CyclophosphamideInduced Hemorrhagic Cystitis with Intravesical Carboprost Tromethamine. The Journal of Urology, 149(4), 719-723.

35. Liem, X., Saad, F., \& Delouya, G. (2015). A Practical Approach to the Management of Radiation-Induced Hemorrhagic Cystitis. Drugs, 75(13), 1471-1482. doi:10.1007/s40265-015-0443-5

36. Liguori, G., Amodeo, A., Mucelli, F. P., Patel, H., Marco, D., Belgrano, E., \& Trombetta, C. (2010). Intractable haematuria: long-term results after selective embolization of the internal iliac arteries. BJU Int, 106(4), 500-503. doi:10.1111/j.1464-410X.2009.09192.x

37. Linder, B. J., Tarrell, R. F., \& Boorjian, S. A. (2014). Cystectomy for refractory hemorrhagic cystitis: contemporary etiology, presentation and outcomes. The Journal of Urology, 192(6), 1687-1692. doi:10.1016/j.juro.2014.06.030

38. Liu, Y. K., Harty, J. I., Steinbock, G. S., Homer, A. H., Goldsetin, D. H., \& Amin, M. (1990). Treatment of Radiation or Cyclophosphamide Induced Hemorrhagic Cystitis using Conjugated Estrogen. The Journal of Urology, 144(1), 41-43.

39. Loffroy, R., Pottecher, P., Cherblanc, V., Favelier, S., Estivalet, L., Koutlidis, N., . . Krause, D. (2014). Current role of transcatheter arterial embolization for bladder and prostate hemorrhage. Diagn Interv Imaging, 95(11), 10271034. doi:10.1016/j.diii.2014.03.008

40. Lojanapiwat, B., Sripralakrit, S., Soonthornphan, S., \& Wudhikarn, S. (2002). Intravesicle Formalin Instillation with a Modified Technique for Controlling Haemorrhage Secondary to Radiation Cystitis. Asian Journal Of Surgery, 25(3), 232-235.

41. Lowe, B. A., \& Stamey, T. A. (1997). Endoscopic topical placement of formalin soaked pledgets to control localized hemorrhage due to radiation cystitis. The Journal of Urology, 158(2), 528-529.

42. Margreiter, M., Sharma, V., Schauer, I., \& Klingler, H. C. (2013). Intravesical hemostatic gelatin matrix instillation in the management of intractable hematuria. Urology, 82(3), 724-728. doi:10.1016/j.urology.2013.06.003 
43. Marks, L. B., Carroll, P. R., Dugan, T. C., \& Anscher, M. S. (1995). The response of the urinary bladder, urethra and ureter to radiation and chemotherapy International Journal of Radiation Oncology*Biology*Physics, 31(5), 1257-1280.

44. McAchran, S. E., \& Rackley, R. R. (2008). Hemorrhagic and Radiation Cystitis Genitourinary Pain And Inflammation. Curent Clinical Urology. : Humana Press.

45. Miller, J., Burfield, G. D., \& Moretti, K. L. (1994). Oral conjugated estrogen therapy for treatment of hemorrhagic cystitis. The Journal of Urology, 151(5), 1348-1350.

46. Moharamzadeh, P., Ojaghihaghighi, S., Amjadi, M., Rahmani, F., \& Farjamnia, A. (2017). Effect of tranexamic acid on gross hematuria: A pilot randomized clinical trial study. Am J Emerg Med, 35(12), 1922-1925. doi:10.1016/j.ajem.2017.09.012

47. Montgomery, B. D., Boorjian, S. A., Ziegelmann, M. J., Joyce, D. D., \& Linder, B. J. (2016). Intravesical silver nitrate for refractory hemorrhagic cystitis. Turk J Urol, 42(3), 197-201. doi:10.5152/tud.2016.38445

48. Moro, J. C., Junior, E. S. B., Riccetto, C. L. Z., \& Palma, P. (2015). Fulminating Hemorrhagic Cystitis: New Trends from Etiology to Treatment. Current Bladder Dysfunction Reports, 10(3), 212-221. doi:10.1007/s11884015-0305-8

49. Nakada, T., Nakada, H., Yoshida, Y., Nakashima, Y., Banya, Y., Fujihira, T., \& Karasawa, K. (2012). Hyperbaric oxygen therapy for radiation cystitis in patients with prostate cancer: a long-term follow-up study. Urologia Internationalis, 89(2).

50. Ouwenga, M., Langston, M., \& Campbell, S. (2004). Use of Fibrin Sealant in Recalcitrant Hemorrhagic Cystitis. The Journal of Urology, 172(4), 13481348. doi:10.1097/01.ju.0000137723.38685.ff

51. Parsons, C. L. (1986). Successful management of radaition cystitis with sodium pentosanpolysulfate. Journal of Urology, 136(4), 813-814.

52. Parsons, C. L. (2011). The role of a leaky epithelium and potassium in the generation of bladder symptoms in interstitial cystitis/overactive bladder, urethral syndrome, prostatitis and gynaecological chronic pelvic pain. BJU Int, 107(3), 370-375. doi:10.1111/j.1464-410X.2010.09843.x

53. Pearce, W. A., Chen, R., \& Jain, N. (2018). Pigmentary Maculopathy Associated with Chronic Exposure to Pentosan Polysulfate Sodium. Ophthalmology, 125(11), 1793-1802. doi:10.1016/j.ophtha.2018.04.026

54. Phelps, K. R., Naylor, K., Brien, T. P., Wilbur, H., \& Haqqie, S. S. (1999). Encephalopathy after Bladder Irrigation with Alum: Case Report and Literature Review. The American Journal of the Medical Sciences, 318(3), 181.

55. Pisco, J. M., Martins, J. M., \& Correia, M. G. (1989). Internal Iliac Artery: Embolization to Control Hemorrhage from Pelvic Neoplasms Radiology, 172(2), 337-339.

56. Pomer, S., Karcher, G., \& Simon, W. (1983). Cutaneous Ureterostomy as Last Resort Treatment of Intractable Haemorrhagic Cystitis Following Radiation. BJU Int, 55(4), 392-394. 
57. Sandhu, S. S., Goldstraw, M., \& Woodhouse, C. R. (2004). The management of haemorrhagic cystitis with sodium pentosan polysulphate. BJU Int, 94(6), 845-847. doi:10.1111/j.1464-410X.2004.05044.X

58. Shao, Y., Lu, G. L., \& Shen, Z. J. (2012). Comparison of intravesical hyaluronic acid instillation and hyperbaric oxygen in the treatment of radiation-induced hemorrhagic cystitis. BJU Int, 109(5), 691-694. doi:10.1111/j.1464-410X.2011.10550.x

59. Singh, I., \& Laungani, M. D. (1992). Intravesical epsilon aminocaproic acid in management of intractable bladder hemorrhage. Urology, 40(3), 227-229.

60. Smit, S., \& Heyn, C. (2010). Management of Radiation Cystitis Nature Reviews Urology, 7, 206-214.

61. Takashi, M., Kondo, A., Kato, K., Murase, T., \& Miyake, K. (1988). Evaluation of intravesical alum irrigation for massive bladder hemorrhage.. Urologia Internationalis, 43(5), 286-288.

62. Talab, S. S., McDougal, W. S., Wu, C. L., \& Tabatabaei, S. (2014). Mucosasparing, KTP laser coagulation of submucosal telangiectatic vessels in patients with radiation-induced cystitis: a novel approach. Urology, 84(2), 478-483. doi:10.1016/j.urology.2014.03.029

63. Thompson, A. A., A; Bahl, A; Borwell, J; Dodds, D; Heath, C; Huddart, R; McNememinm R; Patel, P; Peters, J; Payne, H. (2013). Guidelines for the diagnosis, prevention and management of chemical- and radiation-induced cystitis. . Journal of Clinical Urology, 7(1), 25-35. doi:10.1177/2051415813512647

64. Tirindelli, M. C., Flammia, G. P., Bove, P., Cerretti, R., Cudillo, L., De Angelis, G., . . Rome Transplant, N. (2014). Fibrin glue therapy for severe hemorrhagic cystitis after allogeneic hematopoietic stem cell transplantation. Biol Blood Marrow Transplant, 20(10), 1612-1617. doi:10.1016/j.bbmt.2014.06.018

65. Vasssilis, K., Eftychia, M. A., F. , Ivelina, B. C., A. Dimitrios, C., Nikolaos, K. M., C., \& Argyris, S. (2014). Use of Hyaluronic Acid (Cystistat) for the Treatment of Late Radiation Induced Cystitis in Patients after Prostate Irradiation. Journal of Bioequivalence \& Bioavailability, 06(01). doi:10.4172/jbb.1000174

66. Veerasarn, V., Boonnuch, W., \& Kakanaporn, C. (2006). A phase II study to evaluate WF10 in patients with late hemorrhagic radiation cystitis and proctitis. Gynecol Oncol, 100(1), 179-184. doi:10.1016/j.ygyno.2005.08.014

67. Veerasarn, V., Khorprasert, C., Lorvidhaya, V., Sangruchi, S., Tantivatana, T., Narkwong, L., . . . Ngampanprasert, P. (2004). Reduced recurrence of late hemorrhagic radiation cystitis by WF10 therapy in cervical cancer patients: a multicenter, randomized, two-arm, open-label trial. Radiother Oncol, 73(2), 179-185. doi:10.1016/j.radonc.2004.05.007

68. Weiss, J. P., Mattei, D. M., Neville, E. D., \& Hanno, P. M. (1994). Primary Treatment of Radiation-Induced Hemorrhagic Cystitis with Hyperbaric Oxygen: 10-Year Experience. The Journal of Urology, 151(6), 1514-1517.

69. Westerman, M. E., Boorjian, S. A., \& Linder, B. J. (2016). Safety and efficacy of intravesical alum for intractable hemorrhagic cystitis: A contemporary evaluation. Int Braz J Urol, 42(6), 1144-1149. doi:10.1590/S16775538.IBJU.2015.0588 
70. Wines, M. P., \& Lynch, W. D. (2006). A new minimally invasive technique for treating radiation cystitis: the argon-beam coagulator. BJU Int, 98(3), 610612. doi:10.1111/j.1464-410X.2006.06293.x

71. Zagoria, R. J., Hodge, R. G., Dyer, R. B., \& Routh, W. D. (1993).

Percutaneous Nephrostomy for Treatment of Intractable Hemorrhagic Cystitis. The Journal of Urology, 149(6), 1449-1451.

72. Ziegelmann, M. J., Boorjian, S. A., Joyce, D. D., Montgomery, B. D., \& Linder, B. J. (2017). Intravesical formalin for hemorrhagic cystitis: A contemporary cohort. Can Urol Assoc J, 11(3-4), E79-E82.

doi:10.5489/cuaj.4047 
Figures and Tables

Table 1. Classification of radiation-induced hemorrhagic cystitis

EORTC/RTOG classification

\begin{tabular}{|c|c|c|c|c|}
\hline $\mathbf{1}$ & $\mathbf{2}$ & $\mathbf{3}$ & $\mathbf{4}$ & $\mathbf{5}$ \\
\hline $\begin{array}{c}\text { Slight epithelial } \\
\text { atrophy; minor } \\
\text { telangiectasia; } \\
\text { microscopic } \\
\text { hematuria }\end{array}$ & $\begin{array}{c}\text { Moderate } \\
\text { frequency, } \\
\text { generalized } \\
\text { telangiectasia, } \\
\text { intermittent } \\
\text { macroscopic } \\
\text { hematuria }\end{array}$ & $\begin{array}{c}\text { Severe frequency } \\
\text { and dysuria, } \\
\text { generalized } \\
\text { telangiectasia } \\
\text { (often with } \\
\text { petechiae), } \\
\text { frequent } \\
\text { hematuria with } \\
\text { decreased bladder } \\
\text { capacity }\end{array}$ & $\begin{array}{c}\text { Necrosis/contracted } \\
\text { bladder, severe } \\
\text { hemorrhagic cystitis }\end{array}$ & $\begin{array}{c}\text { Death } \\
\text { directly due } \\
\text { to } \\
\text { hemorrhagic } \\
\text { cystitis }\end{array}$ \\
& & & \\
\hline
\end{tabular}




\section{Table 2. Treatment recommendations}

\section{Initial Assessment and Early Management}

\section{Initial assessment}

- History and Physical

- Laboratory evaluation

- Axial imaging preferably CT Uro

- Cystoscopy +/- fulgerization

if
Presenting with clots \& retention

-Continuous bladder irrigation and hydration

-Inpatient monitoring and transfusion as indicated

-Cystoscopy, clot evacuation +/fulgerization

\section{Intravesical Alum \\ -Inpatient tx in those without renal failure}

or

\section{HBOT}

-Inpatient or

outpatient in stable patients

\section{HA or SPP}

-Outpatient in stable patients, especially those with significant LUTS

* For stable recurrent patients multiple strategies and attempts may be used prior to proceeding with more invasive and irreversible options

\section{Refractory and Life-Threatening Hematuria}

Trans-Arterial Embolization

- preference for selective or superselective embolization Formalin

- limit contact, uptake and reflux
Definitive surgical management - Consideration for urinary diversion with or without cystectomy based on individual patient factors

CT Uro: CT urogram; HA: hyaluronic acid; HBOT: hyperbaric oxygen therapy; SPP: sodium pentosan polysulfate. 